Article

\title{
iso-Petromyroxols: Novel Dihydroxylated Tetrahydrofuran Enantiomers from Sea Lamprey (Petromyzon marinus)
}

\author{
Ke Li ${ }^{1}$, Cory O. Brant ${ }^{1}$, Ugo Bussy ${ }^{1}$, Harshita Pinnamaneni ${ }^{1}$, Hinal Patel ${ }^{1}$, Thomas R. Hoye ${ }^{2}$ \\ and Weiming $\mathrm{Li}$ 1,*
}

1 Department of Fisheries and Wildlife, Michigan State University, East Lansing, MI 48824, USA;

E-Mails: like4@msu.edu (K.L.); brant@msu.edu (C.O.B.); bussy@msu.edu (U.B.); pinnama3@msu.edu (H.P.); patelhi3@msu.edu (H.P.)

2 Department of Chemistry, University of Minnesota, 207 Pleasant Street SE, Minneapolis, MN 55455, USA; E-Mail: hoye@umn.edu

* Author to whom correspondence should be addressed; E-Mail: liweim@msu.edu; Tel: +1-517-432-6705; Fax: +1-517-432-1699.

Academic Editor: Derek J. McPhee

Received: 17 February 2015 / Accepted: 18 March 2015 / Published: 23 March 2015

\begin{abstract}
An enantiomeric pair of new fatty acid-derived hydroxylated tetrahydrofurans, here named iso-petromyroxols, were isolated from sea lamprey larvae-conditioned water. The relative configuration of iso-petromyroxol was elucidated with $1 \mathrm{D}$ and 2D NMR spectroscopic analyses. The ratio of enantiomers $(\mathrm{er})$ in the natural sample was measured by chiral-HPLC-MS/MS to be $c a$. 3:1 of (-)- to (+)-antipodes.
\end{abstract}

Keywords: stereoisomers; relative configuration; cyclostomata

\section{Introduction}

Sea lamprey (Petromyzon marinus) is a useful model for a wide range of research topics, such as chemical communication [1,2], behavior [3,4], neurobiology [5-7], ecology [8], and evolution [9,10]. Its metabolite profile is known to shift through its life stages $[11,12]$. The structural diversity of known sea lamprey metabolites is particularly complex and interesting [4,13-17]. Here we report the relative configuration of two enantiomeric secondary metabolites, which we have named iso-petromyroxol (1) (Figure 1). 


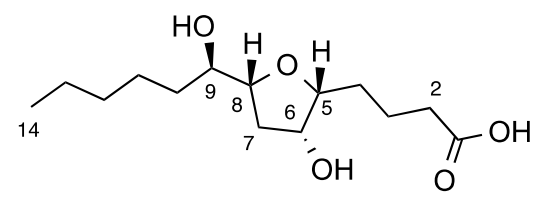

1 iso-petromyroxols $(5 R, 6 R, 8 R, 9 R$ enantiomer is arbitrarily shown)

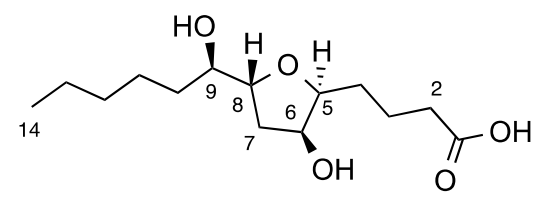

$2(+)$-petromyroxol

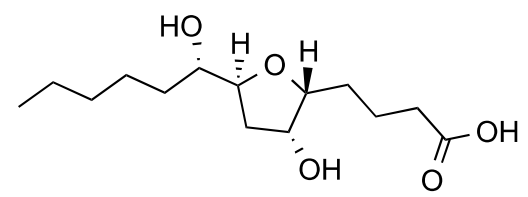

2 (-)-petromyroxol

Figure 1. Structures of iso-petromyroxols (1, this work) and petromyroxols (2, [18]).

In a previous investigation [18], we have described the isolation, structure determination, and preliminary biological activity (olfactory response) of (+)- and (-)-petromyroxols (2). These compounds were isolated from larval sea lamprey-conditioned water. The central tetrahydrofuran (THF) ring in 2 bears a trans-relationship between the two carbon substituents bound to C-5 and C-8. Compounds containing hydroxylated THF subunits have been found in terrestrial plants [19], marine alga [20-22], and marine mollusks [23]. Petromyroxols (2) represent the first examples of dihydroxylated THF-containing metabolites isolated from a vertebrate [18]. Through further studies we have deduced the structures of a related and more minor pair of metabolites, here named iso-petromyroxol (1), which is a diastereomer of $\mathbf{2}$.

\section{Results and Discussion}

Compound 1 was isolated as a colorless oil. High resolution mass spectrometry (HR-MS) signals were observed at $273.1702 \mathrm{Da}\left[(\mathrm{M}-\mathrm{H})^{-}\right.$calculated for $\left.\mathrm{C}_{14} \mathrm{H}_{25} \mathrm{O}_{5}, 273.1707 \mathrm{Da}\right]$ and $297.1678 \mathrm{Da}$ $\left[(\mathrm{M}+\mathrm{Na})^{+}\right.$calculated for $\left.\mathrm{C}_{14} \mathrm{H}_{26} \mathrm{O}_{5} \mathrm{Na}, 297.1672 \mathrm{Da}\right]$ for the negative and positive modes, respectively, of the electrospray ionization spectrum $\left(\mathrm{ESI}^{+/-}\right)$. The $\Delta m / z$ of $0.5 \mathrm{mDa}(1.8 \mathrm{ppm})$ and $0.6 \mathrm{mDa}$ $(2.0 \mathrm{ppm})$ for $[\mathrm{M}-\mathrm{H}]^{-}$and $[\mathrm{M}+\mathrm{Na}]^{+}$suggested $\mathrm{C}_{14} \mathrm{H}_{26} \mathrm{O} \mathrm{O}_{5}$ as the parent formula of compound $\mathbf{1}$.

${ }^{1} \mathrm{H}$ and ${ }^{13} \mathrm{C}-\mathrm{NMR}$ data, in particular the similarity in chemical shifts (Table 1), suggested that compound 1 possessed a structure related to that of the petromyroxols 2 [18]. ${ }^{1} \mathrm{H}-{ }^{1} \mathrm{H}$ COSY analysis indicated that compounds $\mathbf{1}$ and $\mathbf{2}$ shared the same connectivity throughout the central C-2 to C-10 region of the molecules, indicating that $\mathbf{1}$ and $\mathbf{2}$ are diastereomers. A detailed analysis of the NOESY spectrum of 1 revealed correlations between $\mathrm{H}-5 / \mathrm{H}-7 \mathrm{~b}, \mathrm{H}-5 / \mathrm{H}-8$, and $\mathrm{H}-6 / \mathrm{H}-8$ (Figure 2), placing all four of these protons on the same face of the tetrahydrofuran ring. A complementary NOESY correlation was observed between $\mathrm{H}-9 / \mathrm{H}-7 \mathrm{a}$, indicating their cis relationship to one another.
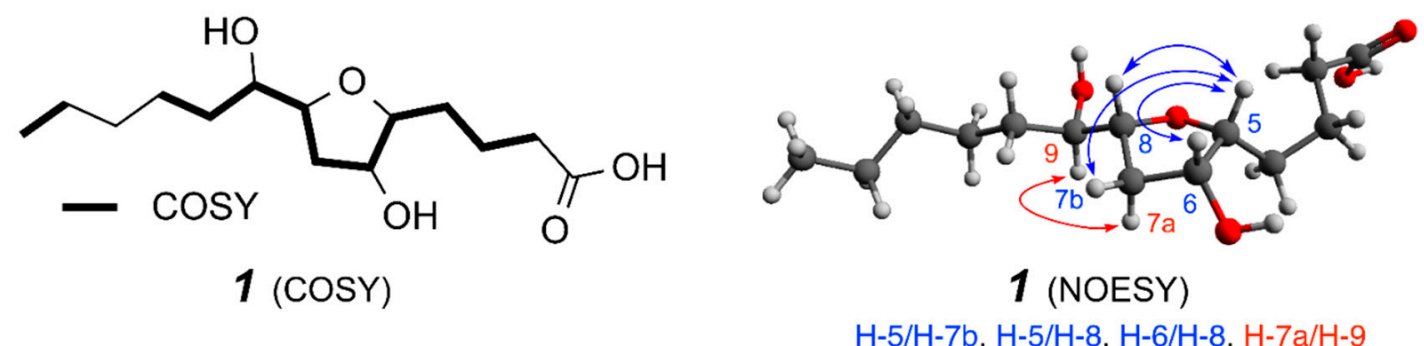

Figure 2. Key COSY and NOESY correlations for iso-petromyroxols (1). 
Table 1. NMR spectroscopic data ( ${ }^{1} \mathrm{H} \mathrm{NMR}, 600 \mathrm{MHz}$ and ${ }^{13} \mathrm{C}-\mathrm{NMR}, 225 \mathrm{MHz}$ in $\left.\mathrm{CDCl}_{3}\right)$ for iso-petromyroxols (1) and petromyroxols [18] (2).

\begin{tabular}{|c|c|c|c|c|}
\hline \multirow{2}{*}{ No. } & \multicolumn{2}{|c|}{ iso-petromyroxols (1) } & \multicolumn{2}{|r|}{ petromyroxols (2) } \\
\hline & $\boldsymbol{\delta}_{\mathrm{C}}$ & $\delta_{\mathrm{H}}$, mult $(J$ in Hz$)$ & $\boldsymbol{\delta}_{\mathbf{C}}$ & $\delta_{\mathrm{H}}$, mult $(J$ in $\mathrm{Hz})$ \\
\hline 1 & 176.5 & - & 177.6 & - \\
\hline 2 & 33.5 & $2.44(\mathrm{t}, 6.7)$ & 33.7 & $2.43 \mathrm{~m}\left(\Sigma J_{\mathrm{S}}=18\right)$ \\
\hline 3 & 21.4 & $1.73 \mathrm{~m}$ & 21.4 & $1.77 \mathrm{~m}, 1.70 \mathrm{~m}$ \\
\hline 4 & 28.0 & $1.72 \mathrm{~m}$ & 28.4 & $1.72 \mathrm{~m}, 1.67 \mathrm{~m}$ \\
\hline 5 & 83.8 & $3.66(\mathrm{ddd}, 2.8,6.2,6.2)$ & 82.5 & 3.79 ddd (ca. $2.5,6.5,6.5)$ \\
\hline 6 & 71.6 & $4.10(\mathrm{dd}, 5.2,2.7)$ & 73.5 & $4.30 \mathrm{dd}(c a .3 .5,3.5)$ \\
\hline $7 \mathrm{a}$ & 38.4 & $1.85(\mathrm{dd} 14.2,3.5)$ & 37.8 & 1.89 ddd $(4.6,9.2,13.7)$ \\
\hline $7 b$ & & $2.39(\mathrm{ddd}, 5.6,9.9,14.0)$ & & $2.02 \mathrm{dd}(6.6,13.4)$ \\
\hline 8 & 79.3 & $3.98(\mathrm{ddd}, 2.2,3.8,10.0)$ & 80.7 & $4.06 \mathrm{ddd}(6.5,6.5,8.9)$ \\
\hline 9 & 73.9 & $3.49(\mathrm{ddd}, 2.4,3.9,8.2)$ & 74.3 & $3.39 \mathrm{~m}\left(\Sigma J_{\mathrm{s}}=18\right)$ \\
\hline 10 & 34.2 & $1.52 \mathrm{~m}$ & 33.3 & $1.40 \mathrm{~m}$ \\
\hline 11 & 25.7 & $1.44 \mathrm{~m}, 1.35 \mathrm{~m}$ & 25.4 & $1.51 \mathrm{~m}, 1.38 \mathrm{~m}$ \\
\hline 12 & 31.7 & $1.28 \mathrm{~m}$ & 32.0 & $1.29 \mathrm{~m}$ \\
\hline 13 & 22.6 & $1.30 \mathrm{~m}$ & 22.8 & $1.31 \mathrm{~m}$ \\
\hline 14 & 14.0 & $0.89 \mathrm{t}(7.0)$ & 14.2 & $0.89 \mathrm{t}(6.9)$ \\
\hline
\end{tabular}

The closest known structural analogues to compound $\mathbf{1}$ are the dihydroxylated THFs 3-5. The first two are natural products, isolated from a brown algae found in Australia [24,25], and the third is a synthetic analog 5 [26]. Differences in the carbon and proton chemical shifts between 1 (Figure 2) and each of 3-5 are shown graphically [27-29] in Figure 3. These comparisons clearly suggest that the best match is between 1 and $\mathbf{4}$, as 4 also possesses an $5 R^{*}, 6 R^{*}, 8 R^{*}, 9 R^{*}$ relative configuration [25]. These results further validated our assignment of the structure of $i s o$-petromyroxols.

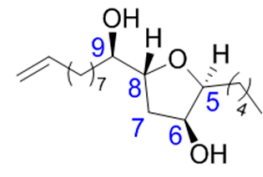

3
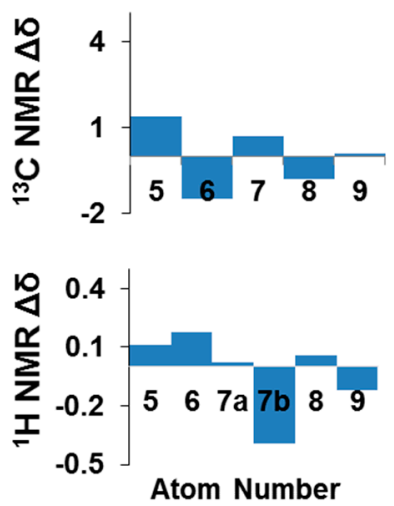

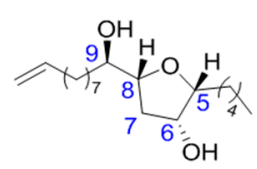

4
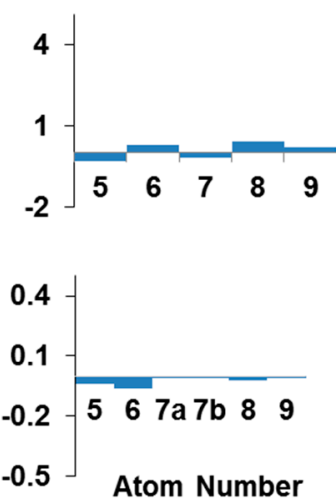

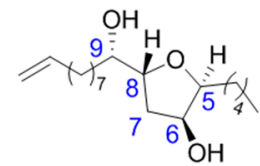

5
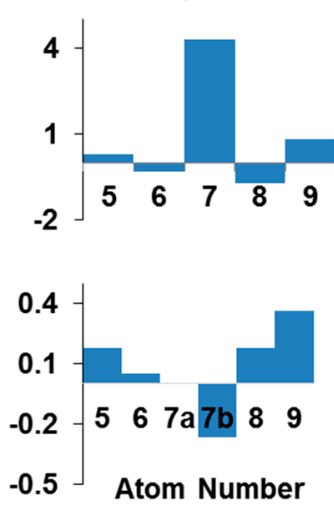

Figure 3. Differences in carbon (upper set of graphs) and proton (lower set of graphs) chemical shifts between iso-petromyroxols (1) and the analogs 3, 4, and 5, respectively, at positions C-5 through C-9 (iso-petromyroxol skeleton numbering used for clarity). 
As was the case for the petromyroxols (2) [18], the natural sample of $\mathbf{1}$ showed a non-zero specific optical rotation $\left([\alpha]_{D}^{25}-5.0, c 0.10, \mathrm{CH}_{3} \mathrm{Cl}\right)$, indicating that the sample of 1 was non-racemic. Analysis by chiral HPLC (Diacel Chiralpak ${ }^{\circledR}$ AD-H, tandem atmospheric-pressure chemical ionization mass spectrometry (APCI-MS/MS detection)) showed that the sample comprised a substantial amount of both enantiomers of 1 (Figure 4). The material was separated to provide $c a .300 \mu \mathrm{g}$ of each antipode. The earlier-eluting enantiomer showed a specific rotation of $[\alpha]_{D}^{25}=+10\left(c 0.20, \mathrm{CHCl}_{3}\right)$, and the latter $[\alpha]_{D}^{25}=-12\left(c 0.20, \mathrm{CHCl}_{3}\right)$. The enantiomeric ratio (er) was $c a .25 \%$ of $(+)-\mathbf{1}$ to $75 \%(-)-\mathbf{1}$ as deduced from peak integration of the chromatogram.

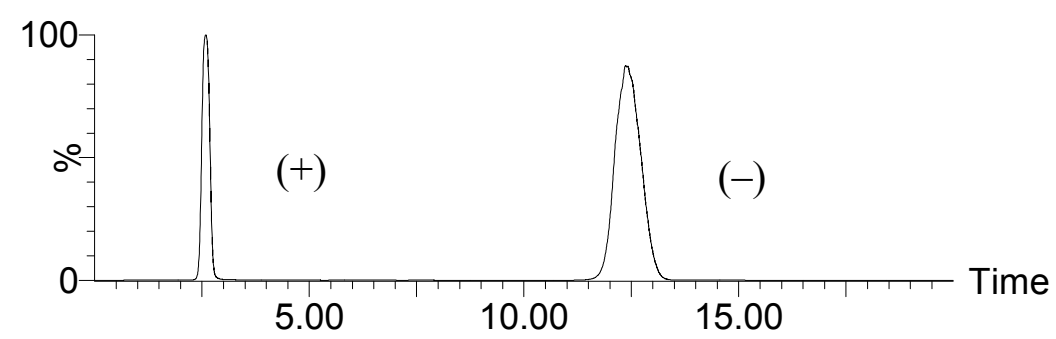

Figure 4. Analysis of (+)- and (-)-iso-petromyroxols by chiral HPLC-MS/MS on APCI positive mode.

\section{Experimental Section}

\subsection{General}

1D and 2D NMR spectra of 1 were recorded on a Bruker $900 \mathrm{MHz}$ or Agilent $600 \mathrm{MHz}$ spectrometer. Mass spectra were measured on a TQ-S TOF LC mass spectrometer (Waters Corporation, Milford, MA, USA). Optical rotation values were determined on a polarimeter model 341 (Perkin Elmer, Waltham, MA, USA) Chromatography supports - silica gel (70-230 and 230-400 mesh), RP-18 reverse-phase silica gel, and Sephadex LH-20—were obtained from E. Merck (Darmstadt, Germany). TLC was performed on glass plates pre-coated with GF254 silica gel, also obtained from E. Merck. Analytes were first visualized with $254 \mathrm{~nm}$ light and then stained with a spray of 5\% anisaldehyde (Sigma-Aldrich, St. Louis, MI, USA) in acidic methanol.

\subsection{Animals}

Procedures involving sea lamprey larvae were conducted in accordance with the Public Health Service (PHS) Policy on Humane Care and Use of Laboratory Animals, incorporated into the Institute for Laboratory Animal Research Guide for Care and Use of Laboratory Animals, and have been approved by the Institutional Animal Use and Care Committee at Michigan State University (Animal use form number: 03/11-053-00). Larval sea lampreys were collected from tributaries of the Laurentian Great Lakes by the Fisheries and Oceans Canada and the US Fish and Wildlife Service according to approved collection permits from the respective government agencies. The larvae were transported to the USA Geological Survey Hammond Bay Biological Station in Millersburg, Michigan, state abbre, USA. The animals were held in flow through tanks ( $0.6 \mathrm{~m}$ deep by $1.8 \mathrm{~m}$ wide by $15.2 \mathrm{~m}$ long). 


\subsection{Extraction of Larval Sea Lamprey Conditioned Water}

Every 4-5 days over the course of $c a .5$ months, sea lamprey larvae-conditioned water $(c a .4000 \mathrm{~L}$ for each cycle), from the tank holding $c a$. 8000-10,000 larvae was passed through four column beds each containing $1 \mathrm{~kg}$ of Amberlite XAD 7HP resin. The flow rate was ca. $200 \mathrm{~mL} / \mathrm{min} / \mathrm{bed}$. Each column was subsequently eluted with $4 \mathrm{~L}$ of methanol at each cycle. The eluent was processed at $40{ }^{\circ} \mathrm{C}$ under reduced pressure using a rotary evaporator, yielding $c a .1 \mathrm{~L}$ solution that comprised primarily water. These solutions were pooled and stored at $-80^{\circ} \mathrm{C}$ until further processing. A $42 \mathrm{~L}$ pool of these combined solutions was thawed and concentrated by lyophilization. The resulting residue was suspended in methanol and filtered through $2 \mu \mathrm{m}$ filter paper to remove the insoluble portion. The filtrate was concentrated again under reduced pressure at $40^{\circ} \mathrm{C}$, yielding $2.0 \mathrm{~g}$ of a dark residue.

\subsection{Purification of iso-Petromyroxol (1)}

The above dark residue was subjected to preparative chromatography over silica gel (150 g) using a gradient of chloroform:methanol, stepped from $10 \%$ to $100 \%$ methanol. Thin layer chromatography (TLC) analysis was used to pool the eluants into nine fractions. Fraction 4, after concentration, yielded ca. $26 \mathrm{mg}$ of a brown oil. This was further purified using two successive Sephadex LH-20 columns that were eluted with $\mathrm{CHCl}_{3}-\mathrm{MeOH}(1: 1)$ and $\mathrm{MeOH}(100 \%)$, respectively. The sample of the non-racemic mixture of iso-petromyroxols $(1,1.4 \mathrm{mg})$ was identified and its purity enrichment judged and guided by TLC.

iso-petromyroxol (1): colorless oil; $[\alpha]_{D}^{25}-5.0$ (c 0.10, MeOH); ${ }^{1} \mathrm{H}$ and ${ }^{13} \mathrm{C}-\mathrm{NMR}$ data, see Table 1; ESI-MS m/z (\%) 273 (3), 193 (5), 168 (5), 145 (7), 143 (14), 129 (100), 127 (25), and 125 (12); HR-ESI-MS m/z 273.1702 (calcd for $\mathrm{C}_{14} \mathrm{H}_{25} \mathrm{O}_{5}, 273.1709[\mathrm{M}-\mathrm{H}]^{-}$).

\subsection{Chiral HPLC-MS/MS Analysis}

A HPLC-MS/MS method was developed to achieve baseline separation of the enantiomers of 1 . These analyses were carried out on a Waters ultra-performance liquid chromatography (ACQUITY UPLC $^{\circledR}$ ) system fitted with a Xevo TQ-S tandem quadruple mass spectrometer (Waters) that used an APCI source in the positive mode. The mixture of 1 was analyzed on a Chiralpak ${ }^{\circledR}$ AD-H column $(2.1 \times 150 \mathrm{~mm}, 5 \mu \mathrm{m})$. Isocratic elution with $n$-hexane:ethanol:formic acid $(85: 15: 0.1, \mathrm{v} / \mathrm{v} / \mathrm{v})$ was performed at a flow rate of $0.70 \mathrm{~mL} / \mathrm{min}$. Multiple reaction monitoring (MRM, 275.0 Da parent and 238.9 Da daughter ions) was performed using optimized cone and collision energy voltages $(23 \mathrm{~V}$ and $10 \mathrm{eV}$, respectively). Data were acquired using MassLynx 4.1 software (Waters). The same method was then used to fractionate the $c a .1 .4 \mathrm{mg}$ sample of the enantiomeric mixture of iso-petromyroxols (1). Two injections of $100 \mu \mathrm{L}$ each were eluted and collected in $c a .40,0.7 \mathrm{~mL}$ fractions. These were then individually analyzed by the above-described HPLC-MS/MS method. The appropriate fractions of each enantiomer were pooled and lyophilized, resulting in (+)-1 $0.3 \mathrm{mg}$ and (-)-1 $0.5 \mathrm{mg}$ of the faster and slower eluting enantiomers, respectively. 


\section{Conclusions}

We have isolated and structurally characterized the iso-petromyroxols (1) and shown that they are diastereomeric with the dihydroxylated tetrahydrofuran-containing petromyroxols (2). The iso-petromyroxols represent a new addition to the molecular diversity of sea lamprey metabolites. The delineation of the absolute configuration of the iso-petromyroxols (1) - e.g., by Mosher ester analysis [18]—will be possible when larger amounts of enantiopure compounds become available.

\section{Supplementary Materials}

Supplementary materials can be accessed at: http://www.mdpi.com/1420-3049/20/03/5215/s1.

\section{Acknowledgments}

We thank Daniel Jones and Lijun Chen of Michigan State University (MSU) Mass Spectrometry Facility as well as Daniel Holmes and Kermit Johnson of MSU NMR Facility for technical assistance. We thank the staff of the Fisheries and Oceans Canada and the USA Fish and Wildlife Service for collection of the sea lamprey larvae used in this study and the staff of the US Geological Survey Hammond Bay Biological Station for their assistance with collection and extraction of water conditioned with sea lamprey larvae. This study was funded by grants from the Great Lakes Fishery Commission.

\section{Author Contributions}

K.L. and W.L. conceived and designed the experiments. K.L., C.O.B., U.B., H.P., and H.P. performed the experiments and analyzed the data. T.R.H. analyzed the data and edited the manuscript. K.L. and W.L. wrote the manuscript. All authors read and approved the final manuscript.

\section{Conflicts of Interest}

The authors declare no conflict of interest.

\section{References}

1. Burnard, D.; Gozlan, R.E.; Griffiths, S.W. The role of pheromones in freshwater fishes. J. Fish Biol. 2008, 73, 1, 1-16.

2. Davidson, Y.W.C.; Huertas, M.; Li, W.M. A Review of Research in Fish Pheromones; Springer: New York, NY, USA, 2011; pp. 467-482.

3. Buchinger, T.J.; Wang, H.; Li, W.; Johnson, N.S. Evidence for a receiver bias underlying female preference for a male mating pheromone in sea lamprey. Proc. R. Soc. B-Biol. Sci. 2013, 280, doi:10.1098/rspb.2013.1966.

4. Li, K.; Brant, C.O.; Siefkes, M.J.; Kruckman, H.G.; Li, W.M. Characterization of a Novel Bile Alcohol Sulfate Released by Sexually Mature Male Sea Lamprey (Petromyzon marinus). PLoS One 2013, doi:10.1371/journal.pone.0068157.

5. Buchanan, J.T. Contributions of identifiable neurons and neuron classes to lamprey vertebrate neurobiology. Prog. Neurobiol. 2001, 63, 441-466. 
6. Laframboise, A.J.; Ren, X.; Chang, S.; Dubuc, R.; Zielinski, B.S. Olfactory sensory neurons in the sea lamprey display polymorphisms. Neurosci. Lett. 2007, 414, 277-281.

7. Ren, X.; Chang, S.; Laframboise, A.; Green, W.; Dubuc, R.; Zielinski, B. Projections from the accessory olfactory organ into the medial region of the olfactory bulb in the sea lamprey (Petromyzon marinus): A novel vertebrate sensory structure? J. Comp. Neurol. 2009, 516, 105-116.

8. Treble, A.J.; Jones, M.L.; Steeves, T.B. Development and evaluation of a new predictive model for metamorphosis of Great Lakes larval sea lamprey (Petromyzon marinus) Populations. J. Great Lakes Res. 2008, 34, 404-417.

9. Kavanaugh, S.I.; Nozaki, M.; Sower, S.A. Origins of gonadotropin-releasing hormone $(\mathrm{GnRH})$ in vertebrates: Identification of a novel GnRH in a basal vertebrate, the sea lamprey. Endocrinology 2008, 149, 3860-3869.

10. Sower, S.A.; Moriyama, S.; Kasahara, M.; Takahashi, A.; Nozaki, M.; Uchida, K.; Dahstrom, J.M.; Kawauchi, H. Identification of sea lamprey GTH beta-like cDNA and its evolutionary implications. Gen. Comp. Endocrinol. 2006, 148, 1, 22-32.

11. Lowartz, S.; Petkam, R.; Renaud, R.; Beamish, F.W.H.; Kime, D.E.; Raeside, J.; Leatherland, J.F. Blood steroid profile and in vitro steroidogenesis by ovarian follicles and testis fragments of adult sea lamprey, Petromyzon marinus. Comp. Biochem. Phys. A 2003, 134, 365-376.

12. Wilkie, M.P.; Claude, J.F.; Cockshutt, A.; Holmes, J.A.; Wang, Y.S.; Youson, J.H.; Walsh, P.J. Shifting patterns of nitrogen excretion and amino acid catabolism capacity during the life cycle of the sea lamprey (Petromyzon marinus). Physiol. Biochem. Zool. 2006, 79, 885-898.

13. Li, K.; Brant, C.O.; Huertas, M.; Hur, S.K.; Li, W. Petromyzonin, a hexahydrophenanthrene sulfate isolated from the larval sea lamprey (Petromyzon marinus L.). Org. Lett. 2013, 15, 5924-5927.

14. Li, W.M.; Scott, A.P.; Siefkes, M.J.; Yan, H.G.; Liu, Q.; Yun, S.S.; Gage, D.A. Bile acid secreted by male sea lamprey that acts as a sex pheromone. Science 2002, 296, 138-141.

15. Hoye, T.R.; Dvornikovs, V.; Fine, J.M.; Anderson, K.R.; Jeffrey, C.S.; Muddiman, D.C.; Shao, F.; Sorensen, P.W.; Wang, J. Details of the structure determination of the sulfated steroids PSDS and PADS: New components of the sea lamprey (Petromyzon marinus) migratory pheromone. J. Org. Chem. 2007, 72, 7544-7550.

16. Sorensen, P.W.; Fine, J.M.; Dvornikovs, V.; Jeffrey, C.S.; Shao, F.; Wang, J.Z.; Vrieze, L.A.; Anderson, K.R.; Hoye, T.R. Mixture of new sulfated steroids functions as a migratory pheromone in the sea lamprey. Nat. Chem. Biol. 2005, 1, 324-328.

17. Li, K.; Siefkes, M.J.; Brant, C.O.; Li, W. Isolation and identification of petromyzestrosterol, a polyhydroxysteroid from sexually mature male sea lamprey (Petromyzon marinus L.). Steroids 2012, 77, 806-810.

18. Li, K.; Huertas, M.; Brant, C.; Chung-Davidson, Y.-W.; Bussy, U.; Hoye, T.R.; Li, W. (+)-and (-)Petromyroxols: Antipodal tetrahydrofurandiols from larval sea lamprey (Petromyzon marinus L.) that elicit enantioselective olfactory responses. Org. Lett. 2014, doi:10.1021/o15033893.

19. Jiang, Z.; Chen, R.Y.; Chen, Y.; Yu, D.Q. Donnaienin, a new acetogenin bearing a hydroxylated tetrahydrofuran ring. J. Nat. Prod. 1998, 61, 86-88.

20. Cueto, M.; Darias, J. Uncommon tetrahydrofuran monoterpenes from Antarctic Pantoneura plocamioides. Tetrahedron 1996, 52, 5899-5906. 
21. Oztunc, A.; Imre, S.; Lotter, H.; Wagner, H. 2 C-15 Bromoallenes from the red alga laurencia-obtusa. Phytochemistry 1991, 30, 255-257.

22. Ji, N.Y.; Li, X.M.; Xie, H.; Ding, J.; Li, K.; Ding, L.P.; Wang, B.G. Highly oxygenated triterpenoids from the marine red alga Laurencia mariannensis (Rhodomelaceae). Helv. Chim. Acta 2008, 91, 1940-1946.

23. Manzo, E.; Gavagnin, M.; Bifulco, G.; Cimino, P.; di Micco, S.; Ciavatta, M.L.; Guo, Y.W.; Cimino, G. Aplysiols A and B, squalene-derived polyethers from the mantle of the sea hare Aplysia dactylomela. Tetrahedron 2007, 63, 9970-9978.

24. Warren, R.; Wells, R.; Blount, J. A novel lipid from the brown alga Notheia anomala. Aust. J. Chem. 1980, 33, 891-898.

25. Capon, R.J.; Barrow, R.A.; Rochfort, S.; Jobling, M.; Skene, C.; Lacey, E.; Gill, J.H.; Friedel, T.; Wadsworth, D. Marine nematocides: Tetrahydrofurans from a southern Australian brown alga, Notheia anomala. Tetrahedron 1998, 54, 2227-2242.

26. Wang, Z.M.; Shen, M. Enantiocontrolled construction of functionalized tetrahydrofurans: Total synthesis of $(6 S, 7 S, 9 R, 10 R)-6,9$-epoxynonadec-18-ene-7,10-diol, a marine natural product. J. Org. Chem. 1998, 63, 1414-1418.

27. Kobayashi, Y.; Lee, J.; Tezuka, K.; Kishi, Y. Toward creation of a universal NMR database for the stereochemical assignment of acyclic compounds: The case of two contiguous propionate units. Org. Lett. 1999, 1, 2177-2180.

28. Benowitz, A.B.; Fidanze, S.; Small, P.; Kishi, Y. Stereochemistry of the core structure of the mycolactones. J. Am. Chem. Soc. 2001, 123, 5128-5129.

29. Kobayashi, Y.; Tan, C.-H.; Kishi, Y. Toward creation of a universal NMR database for stereochemical assignment: Complete structure of the desertomycin/oasomycin class of natural products. J. Am. Chem. Soc. 2001, 123, 2076-2078.

Sample Availability: Samples of the compounds iso-petromyroxols are unavailable from the authors.

(C) 2015 by the authors; licensee MDPI, Basel, Switzerland. This article is an open access article distributed under the terms and conditions of the Creative Commons Attribution license (http://creativecommons.org/licenses/by/4.0/). 